\title{
TOF-LIDAR signal processing using the CFAR detector
}

\author{
Takashi Ogawa ${ }^{1,2}$ and Gerd Wanielik ${ }^{1}$ \\ ${ }^{1}$ Professorship of Communication Engineering, Chemnitz University of Technology, Chemnitz, Germany \\ ${ }^{2}$ DENSO Corporation, Kariya, Japan
}

Correspondence to: Takashi Ogawa (takashi.ogawa@s2014.tu-chemnitz.de, takasi.ogawa@denso.co.jp)

Received: 15 January 2016 - Accepted: 30 August 2016 - Published: 28 September 2016

\begin{abstract}
In recent years, the lidar sensor has been receiving greater attention as being one of the prospective sensors for future intelligent vehicles. In order to enable advanced applications in a variety of road environments, it has become more important to detect various objects at a wider distance. Therefore, in this research we have focused on lidar signal processing to detect low signal-to-noise ratio (SNR) targets and proposed a higher sensitive detector. The detector is based on the constant false alarm rate (CFAR) processing framework in which an additional functionality of adaptive intensity integration is incorporated. Fundamental results through static experiments have shown a significant advantage in the detection performance in comparison to a conventional detector with constant thresholding.
\end{abstract}

\section{Introduction}

In hopes of achieve safe, comfortable, and convenient vehicle transportation in the near future, the highly automated driving or autonomous driving systems have been receiving greater attention in recent years. The research has attracted increased attention and has been accelerated by the DARPA (Defense Advanced Research Projects Agency) Urban Challenge, where the fundamental experimental vehicles equipped with special devices and software have been prototyped by participants mainly from research institutes (Levinson et al., 2011). Moreover, several advanced car manufactures and innovative IT companies have evolved the technologies and implemented them in their prototype vehicles, which have been driven on privileged parts of public roads in order to investigate their functionality and capability before introducing them into the market (Ziegler et al., 2014a). As such the automated system basically consists of several subfunctions such as environment perception, behavior plan- ning, and vehicle control. Among them, the perception of ego-vehicle surroundings is a crucially important task in the front-end processing of the whole system, by which accurate and robust $360^{\circ}$ perception is required and approached by integrating several types of electro-magnetic sensors, a global navigation satellite system (GNSS), and precise digital maps (Ziegler et al., 2014b; Dickmann et al., 2015).

The important role of the sensors is to detect dynamic objects or events that have not been registered in the digital map beforehand, and the scope of the objects is expected to be enlarged according to the functional advancement and the expansion of the application filed. For example, temporary existing on-road objects (e.g., tires, boxes), which are in most cases left by other traffic participants who have traveled on the road before, are going to be in the next scope. In order to detect such wide variations of objects at a significantly wider distance, sensors are required to improve detection sensitivity; therefore, various technologies are being developed in the field of automotive lidar technology.

The straight-forward approach is to improve the detection sensitivity of optical devices and systems on the hardware that high power (with eye-safe) laser diodes, high sensitive photo detectors, and advanced scanning systems are being developed. These hardware technologies contribute to enlarging the received intensity from the detection target and also decrease the background noise in the signal, which means the signal-to-noise ratio (SNR) is improved. In general terms, this innovation has a high potential to achieve significant improvements with a low negative impact on the cost and dimensions of the hardware in the future.

The other approach is the signal processing, which deals with the sensor raw data before the point measurements. The common technique used to increase the SNR is digital filtering. If the frequency characteristics of the echo signal and background noise are significantly decoupled, a properly de- 
Table 1. Basic specification of lidar.

\begin{tabular}{ll}
\hline Terms & Specifications \\
\hline Detection range & $40 \mathrm{~m}$ at $10 \%$ reflectivity \\
\hline Field of view & $\begin{array}{l}\text { Azimuth: } 40^{\circ} \\
\text { Elevation: } 8^{\circ}\end{array}$ \\
\hline Angular resolution & $\begin{array}{l}\text { Azimuth: } 0.1^{\circ} \\
\text { Elevation: } 1.0^{\circ}\end{array}$ \\
\hline Update frequency & $10 \mathrm{~Hz}$ \\
\hline
\end{tabular}

signed low-pass filter or band-pass filter enables one to increase the SNR. Furthermore, the coherent integration is also known as an effective method that enhances the SNR by integrating multiple signals derived from an identical target in the spatial or temporal domain. This integration performs effectively if the sensor has significantly high spatial or temporal resolution.

Furthermore, on the final stage of signal processing, a thresholding is applied to the raw data to generate point measurements that represent the existence of an existing target and its corresponding distance from the sensor. Since the detector is expected to achieve a high detection rate of the target with a low false detection rate, the threshold is required to be adjusted at a proper level considering the state of the background environment. The constant false alarm rate (CFAR) detector is a well-known effective approach for such a problem, and has been applied to automotive radar applications (Rohling and Mende, 1996). It is a method of adaptive thresholding by which the background uncertainty is dynamically calculated, so that it improves detection performance on a lower SNR target by applying the proper thresholding.

In this paper, we discuss the signal processing for lidar sensors, which especially detects objects with time-offlight (TOF) measurements, whose brief explanation is written in Sect. 2. Section 3 describes the basic CFAR algorithm, and an algorithm developed in this research, which unifies the functionality of signal integration with background estimation in the CFAR framework is presented in Sect. 4. The performance evaluation of the developed detector using real sensor data is described in Sect. 5. Finally, this paper is concluded in Sect. 6 with the summary and future plans for this research.

\section{Lidar}

A basic specification of the lidar used in this research is listed in Table 1. It is an automotive lidar prototyped by DENSO Corporation, which is supposed to be utilized in various applications for advanced vehicle safety, e.g., pedestrian detection and tracking (Ogawa et al., 2011). The lidar emits laser

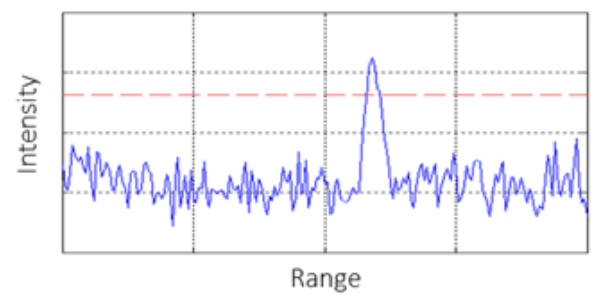

Figure 1. Example of raw intensity measurement and detection threshold at single bearing (solid blue line: intensity; dashed red line: threshold).

beams into the vehicle's surroundings with two-dimensional (2-D) scanning with 401 azimuth and 8 elevation angles. It detects objects that may exists, and measures the distance and intensity in each bearing based on the time-of-flight principle. If the sensor is installed inside the vehicle cabin behind a windshield with $5 \%$ transmittance, the detection range of a target with $10 \%$ reflectivity is about $40 \mathrm{~m}$.

During the observation at each bearing, the received intensity is temporally sampled by an analog-to-digital converter (ADC) with a certain time interval. The sets of the sampled intensities at every scan bearing are stored in an internal memory as raw data. Figure 1 shows an example of the raw data (solid blue line) at a certain bearing together with a conventional detection threshold (dashed red line), which declares the existence of an object. The intensity value on the vertical axis is digitized by the ADC after its offset bias is compensated. The threshold is consistent over all range bins, which is dynamically adjusted at every scan bearing in order to control the false alarm rate by analyzing its background noise intensities. Assuming the probability density function of the noise to be an ideally zero mean Gaussian distribution, its standard deviation is calculated and utilized to set the threshold. In this paper, the threshold is set at the level corresponding to 5 times the standard deviation above ground level, where the probability of false alarm at every sampled point of intensity is theoretically on the order of $10^{-5}$. This constant-thresholding method is referred to as the conventional detector for performance evaluation in Sect. 5 .

Here the physical process of the observation is explained by the lidar equation. The basic form of the equation is

$P(r)=C \frac{\beta(r)}{r^{n}} \exp (-2 \tau)$,

where $P(r)$ is the intensity of optical energy received from a target at range $r, \beta(r)$ is the backscatter coefficient, and $\tau$ is the optical thickness. $C$ is a constant depending on the lidar system determined by the transmitting laser power, pulse duration, optical efficiency of the receiver, etc.; $n$ is an integer between 2 and 4 , which is determined by the geometrical relationship between the dimension of the emitted laser beam and the cross section of the target. If the beam size at the target distance is sufficiently larger than the cross section in 


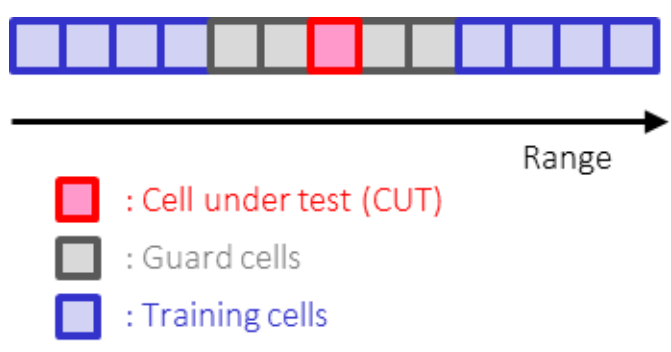

Figure 2. Basic configuration of CFAR sliding operator.

both dimensions of azimuth and elevation, it is set to 4 , and if the beam size is smaller in both dimensions, it is set to 2; otherwise, it is determined to be 3. Equation (1) represents the dynamics of the received intensity in inverse proportion to the $n$th power of the distance to the target, and therefore it links the increase on detection range and SNR improvement.

\section{CFAR algorithms}

The CFAR algorithm is widely used in modern radar signal processing. It enables an adaptive thresholding by learning the state of the background, so that it suitably works in clutter, interference, or other uncertain environments. The threshold adaptation is performed by applying a cell operator to the sensor raw data based on a sliding window technique. The basic design of the operator consists of a cell under test (CUT), guard cells, and training cells, where each cell corresponds to a certain range bin on which the intensity is discretely sampled by ADC. A basic configuration of the operator is shown in Fig. 2.

The CUT is a point where the target existence is evaluated. The training cells refer to the intensities around the CUT in order to locally and statistically evaluate the background level. Based on these background intensities, a single or multiple representative parameter of the background is calculated, for example, the average (e.g., mean) value or rank-ordered value (e.g., median) of the intensities. The former is called cell averaging CFAR (CA-CFAR) and the latter is referred as ordered statistic CFAR (OS-CFAR), whereas the OS-CFAR is generally recognized as the more robust technique in multiple-target situations (Rohling, 2011). Furthermore, other derivations and combinations to achieve better background estimation with lower computational effort have been investigated (Cao et al., 2010; Rohling, 2013). The remaining guard cells work as a range margin in order that the intensities of training cells are not affected by an existing object at around CUT. Then, the detection threshold is determined above the adaptively estimated background level at each point, and its procedure is generally classified into two types. One is a parametric determination of the threshold based on the statistical characteristics of the background, and the other is a non-parametric by which the threshold is

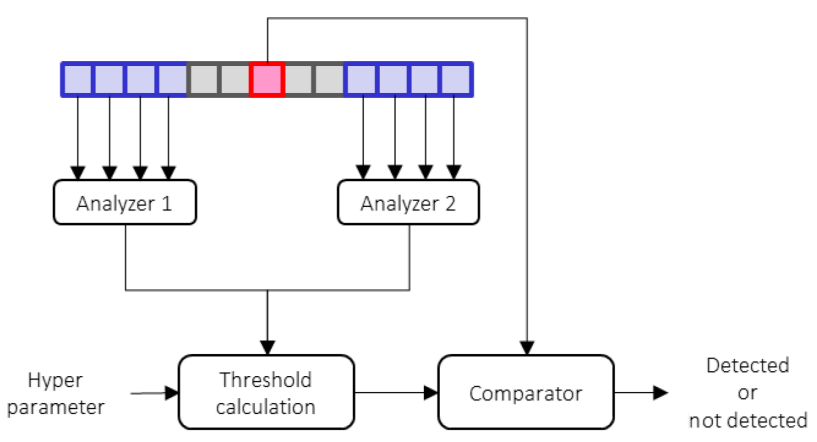

Figure 3. General configuration of CFAR processing.

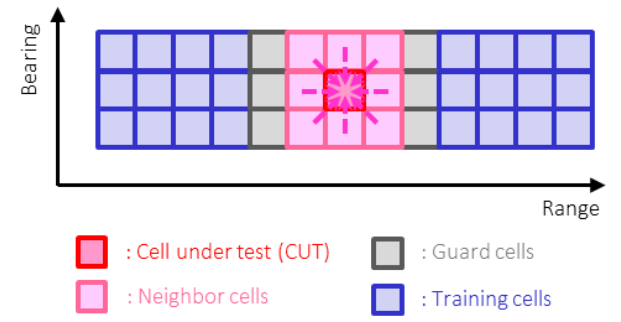

Figure 4. Extended operator with neighbor cells for intensity integration.

experimentally determined. The general configuration of the CFAR processing is illustrated in Fig. 3.

\section{The extended CFAR detector}

Aiming to improve the detection performance on a low-SNR target, we propose an extended CFAR detector that enables one to incorporate an additional functionality of intensity integration. In order to implement this additional processing on the CFAR framework, the sliding cell operator is extended with supplemental neighbor cells, as shown in Fig. 4. The neighbor cells are added around the CUT in order to specify the region of the intensity integration, where the size is determined depending on the situation. Moreover, a positive numerical value is assigned to every cell of the region, which determines the weight of the integration. Then, the intensity after the weighted integration at the CUT is evaluated in an orderly manner with the CFAR adaptive thresholding. This extended processing is applied in both the range and bearing domain; therefore, the operator forms a 2-D window.

\subsection{Range domain processing}

One of the physical characteristics on raw intensity measurements of the lidar used in this research is that the temporal pulse width of a target echo is correlated to its amplitude, where in general the pulse width expands broader as the amplitude becomes higher. Therefore, focusing on this phenomenon, the intensity is integrated over neighboring 


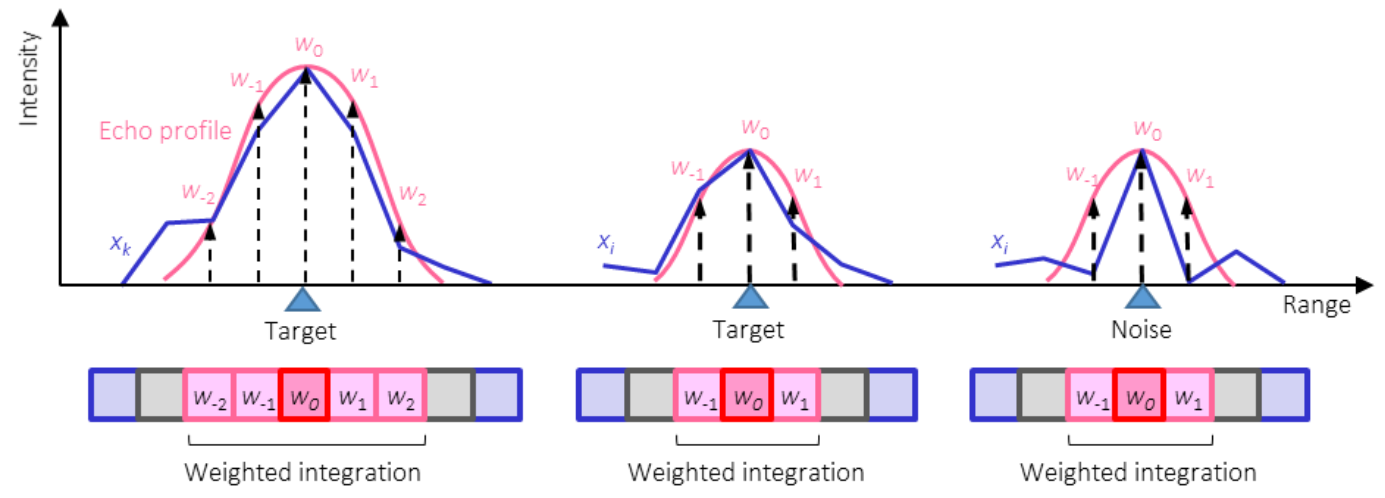

Figure 5. Intensity integration in range domain.

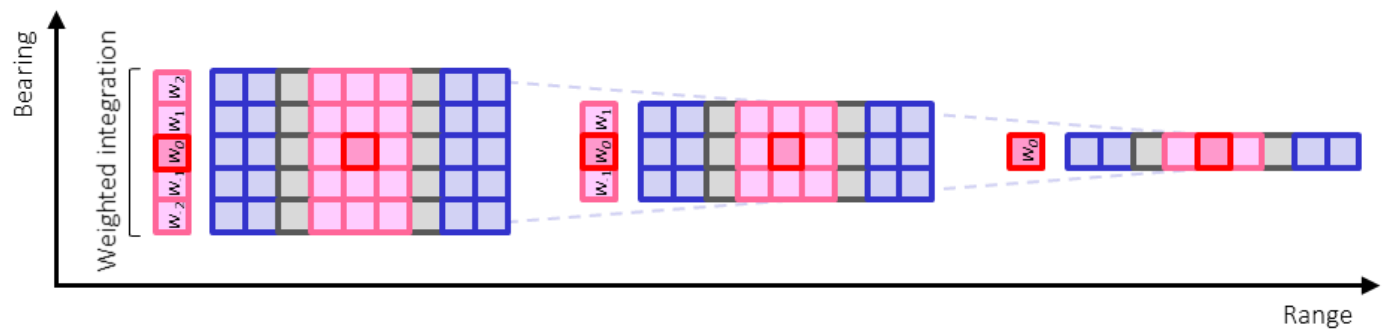

Figure 6. Intensity integration in bearing domain.

range bins, depending on the intensity at the CUT, as shown in Fig. 5.

The number of range bins for integration, which corresponds to the sum of the CUT and neighbor cells, is calculated with the following equation:

$N^{\text {range }}= \begin{cases}N_{\text {minge }}^{\text {range }} & \text { if } i_{\text {CUT }} \leq I_{\text {th }}, \\ N_{\text {min }}^{\text {range }}+2 \cdot \operatorname{ceiling}\left(\log _{10}\left(i_{\text {CUT }} / I_{\text {th }}\right)\right) & \text { else }\end{cases}$

where $i_{\text {CUT }}$ is an intensity measurement at the CUT, and $I_{\text {th }}$ is a conventional detection threshold described in Sect. 2 . $N_{\text {min }}^{\text {range }}$ is an odd integer that defines a minimum number of range bins for the integration, which is set to 7 in Sect. 5 for performance evaluation. Next, the integration weighting factor at every bin is calculated according to an expected intensity derived from the target echo profile model in the following equations:

$\bar{w}_{k_{\mathrm{r}}}^{\mathrm{range}}=w_{k_{\mathrm{r}}}^{\text {range }} / \sum_{k_{\mathrm{r}}} w_{k_{\mathrm{r}}}^{\text {range }}$,

$w_{k_{\mathrm{r}}}^{\text {range }}=\frac{1}{\sqrt{2 \pi}} \exp \left\{-\frac{1}{2} \cdot\left(\frac{k_{\mathrm{r}}}{\left(\left(N^{\text {range }}-1\right) / 2\right)} d\right)^{2}\right\}$.

$k_{\mathrm{r}}$ is an integer value of the range bin index between $\pm\left(N^{\text {range }}-1\right) / 2$, which takes 0 if the bin corresponds to the CUT and a non-zero value for the neighboring range bin around the CUT. Equation (4) represents the echo profile model based on Gaussian shape, where $d$ is a positive tuning parameter that defines a degree of the pulse extent in the range domain. This integration process nearly works as model-based filtering, which enables one to emphasize the intensity at the CUT if the target exists, otherwise it suppresses the noise intensity if no target exists.

\subsection{Bearing domain processing}

The integration processing over neighbor bearing bins is based on the geometrical relationship between the spatial resolution of the sensor observation and dimension of the relevant target for each application. Indeed, given a certain minimum size of the target, several coherent signals are obtained at multiple bearings if the target is detected as an extended target when it exists at a certain scope of the range. Thus, as shown in Fig. 6, the intensity integration is carried out over these bearings when the coherent intensities from the target are potentially available.

Here the number of bearings where the intensities are integrated is dependent on the range of the CUT, which is calculated in the following equations:

$$
\begin{aligned}
& N^{\text {bearing }}= \begin{cases}1 & \text { if } n \leq 1 \\
N_{\max }^{\text {bearing }} & \text { if } n \geq N_{\max }^{\text {bearing }} \\
\text { floor }(n)+1 & \text { if } 1<n<N_{\max }^{\text {bearing }} \& \text { floor }(n) \bmod 2=0 \\
\text { floor }(n)+2 & \text { if } 1<n<N_{\max }^{\text {bearing }} \& \text { floor }(n) \bmod 2 \neq 0\end{cases} \\
& n=\frac{w}{r \cdot \Delta \theta} .
\end{aligned}
$$

$n$ is a potential number of the bearings that the target could exist in front, $r$ is the range of the CUT, $\Delta \theta$ is the azimuth angle resolution of the sensor observation, and $w$ is a minimum 




Figure 7. Example of calculation results by conventional (upper panel) and extended detector (lower panel).

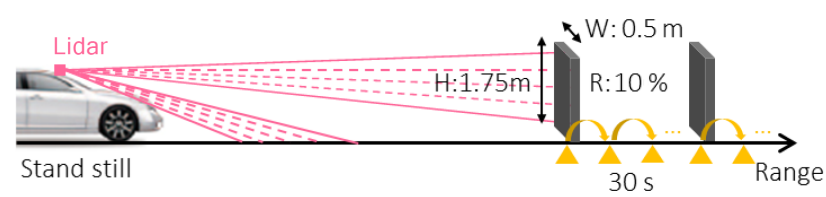

Figure 8. Experimental setup.

width of the assuming target, which is set to 0.3 in the following evaluation. $N_{\max }^{\text {bearing }}$ is an odd integer, which defines the maximum number of bearings for integration, which is set to 5 . Then the corresponding weighting factor of the integration $\bar{w}_{k}^{\text {bearing }}$ is calculated with the following equations:

$\bar{w}_{k_{\mathrm{b}}}^{\text {bearing }}=w_{k_{\mathrm{b}}}^{\text {bearing }} / \sum_{k_{\mathrm{b}}} w_{k_{\mathrm{b}}}^{\text {bearing }}$,

$w_{k_{\mathrm{b}}}^{\text {bearing }}= \begin{cases}(n-\text { floor }(n)) / 2 & \text { if }\left|k_{\mathrm{b}}\right|=\left(N^{\text {bearing }}-1\right) / 2 . \\ 1 & \text { else }\end{cases}$

$k_{\mathrm{b}}$ is an integer value of the bearing bin index between $\pm\left(N^{\text {bearing }}-1\right) / 2$, which takes 0 if the bin corresponds to the CUT and a non-zero value for neighboring bearing bin around the CUT.

\subsection{Decision-making}

An example of the calculation results by the extended detector in comparison to the conventional constant thresholding is shown in Fig. 7.

In the upper chart, the raw intensity measurement (solid blue) and its conventional detection threshold (dashed red line) are shown, and in the lower chart, the intensity after the integration (solid blue), the adaptive threshold (dashed red) as well as the calculated baseline of the background (dashed light blue) are represented. Note that the red arrow means a target is detected, where its range cell and intensity indices are the numbers in the bracket. Comparing the results between before and after the integration, a certain degree of SNR improvement is recognized, which is remarkably seen

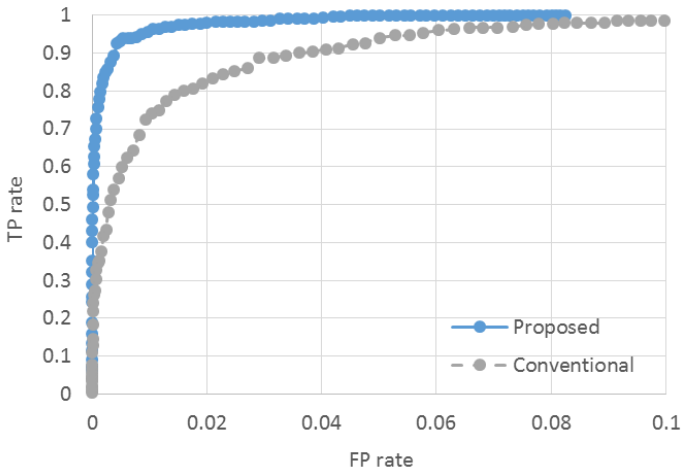

Figure 9. Example of ROC curves (blue: proposed, gray: conventional).

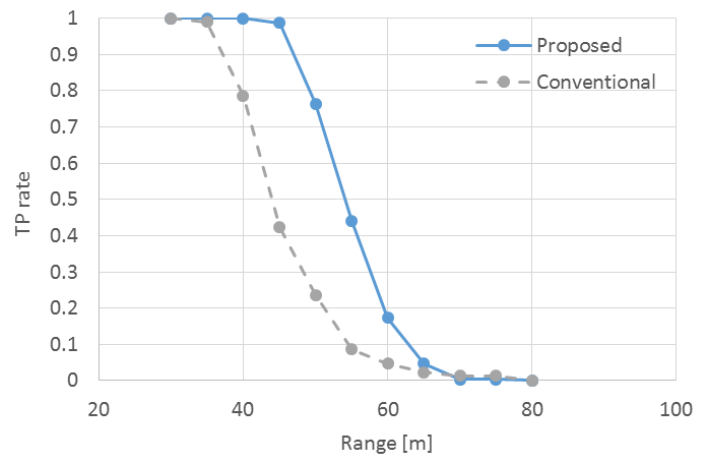

Figure 10. Comparison on TP rate (blue: proposed, gray: conventional).

around the target. With regard to the manner of the threshold determination for the extended detector, the parametric approach mentioned in Sect. 3 is used. Given a standard deviation of the background noise on the raw intensity as well as the integration weighting factors calculated by Eqs. (3) and (7), the substitute standard deviation of the noise after the integration is calculated with the following equations:

$\sigma^{\prime}=\sqrt{\sum_{k_{\mathrm{r}}, k_{\mathrm{b}}}\left(\bar{w}_{k_{\mathrm{r}}, k_{\mathrm{b}}} / \sum_{k_{\mathrm{r}}, k_{\mathrm{b}}} \bar{w}_{k_{\mathrm{r}}, k_{\mathrm{b}}}\right)^{2} \sigma}$,
$\bar{w}_{k_{\mathrm{r}}, k_{\mathrm{b}}}=\bar{w}_{k_{\mathrm{r}}}^{\text {range }} \cdot \bar{w}_{k_{\mathrm{b}}}^{\text {bearing }}$,

where $\sigma$ is the standard deviation of the raw intensity, $\bar{w}_{k_{\mathrm{r}}}$ and $\bar{w}_{k_{\mathrm{b}}}$ is the integration weighting factor at the neighbor bin index of $k_{\mathrm{r}}$ and $k_{\mathrm{b}}$. Therefore, the threshold is set above the background baseline at the level corresponding to 5 times of the standard deviation $\sigma^{\prime}$. Thus, the adaptive threshold is determined depending on the integration process and it generally becomes larger as the distance increases. The number of bearings of the integration is decreased at further distance, while the number of range cells integrated is also smaller, since the target intensity becomes lower. 


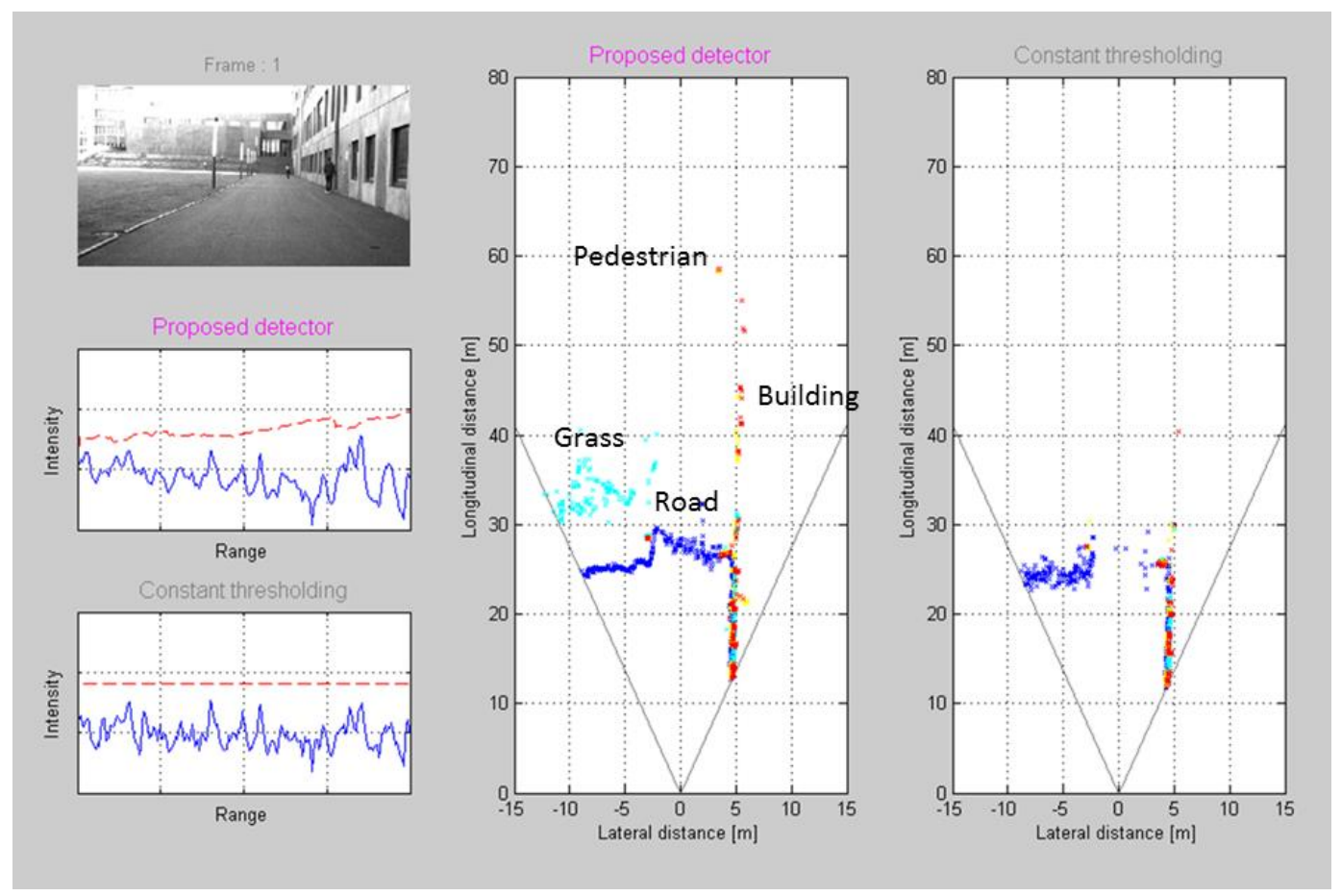

Figure 11. Example of point measurements (middle chart: proposed; right chart: conventional).

\section{Evaluation}

We have evaluated the performance of the proposed detector, described in Sect. 4, by comparing to the conventional constant-thresholding detector, mentioned in Sect. 2.

\subsection{Setup}

The experimental setup is shown in Fig. 8. The test vehicle is equipped with the lidar behind the windshield inside the cabin and remains stationary during the experiment. The detection target is about $0.5 \mathrm{~m}$ wide and $1.75 \mathrm{~m}$ high with $10 \%$ diffuse reflection in the near-infrared band, which is utilized in this lidar. The target is placed in front of the vehicle at $5 \mathrm{~m}$ intervals and is observed for $30 \mathrm{~s}$ at each point. The collected raw data are processed by both the conventional and proposed detector, and these results are compared.

\subsection{Results}

At first, we compare the receiver operating characteristics (ROC) curves of both detectors by handling the detection threshold parameters. Here, we define the true positive (TP) rate as a ratio between the number of data frames in which the target is detected and the total number of the data frames. Meanwhile, the false positive (FP) rate is a ratio between the number of range bins with false detection and the number of bins where no target exists, which is based on whole data frames. Specifically, the total number of range bins behind the target in all the data frames is the denominator for the FP calculation. Additionally, note that only one bearing, where the target is present in front, is considered for both the TP and FP calculation. Subsequently, based on these definitions, Fig. 9 shows the ROC on the condition that the target stands at $60 \mathrm{~m}$ distance, where the conventional detector does not significantly detect the target because of degrading SNR on the observed intensity. The difference of two curves is clearly recognized, which means that the detection performance is significantly improved by the proposed detector.

Next, we have set the threshold parameters as both the detectors have almost the same FP rate of $10^{-5}$, and then the TP rate has been respectively calculated at every target distance. Figure 10 shows the results, where the detection rate of both detectors at closer than $35 \mathrm{~m}$ is nearly 1 and gradually degrades with further distance. Comparing the range with TP rate of 0.8 , for example, it is extended from approximately 40 to $50 \mathrm{~m}$. According to the lidar equation (Eq. 1), this improvement is interpreted as an approximately $50 \%$ lower SNR target becomes detectable by the proposed approach.

Finally, both the detectors are compared on point measurements after the thresholding. Figure 11 visualizes an example of the point measurements with a bird's-eye view, together with the charts of intensity and threshold for both the detectors at the same bearing as well as the corresponding forward image. The color of the points in the bird's-eye view corresponds to the bearing of the elevation scan, and, for reasons of better visibility, only the measurements of the upper four bearings are plotted. By comparing the number of the mea- 
surements on both views, the proposed detector produces a larger number of measurements and almost no false detection appeared in this scene. In particular, a significant improvement is recognized in the measurements of the walking pedestrian, the wall of the building, the road surface, and grass, which have become detectable at a wider distance or are newly available by the proposed detector.

\section{Conclusions}

In this paper, we have discussed lidar signal processing to detect a low SNR target. The developed detector works in the CFAR framework and is extended by incorporating an additional functionality of adaptive signal integration. The results of fundamental experiments have shown a significant improvement on the detection performance, which leads to obtain further point measurements.

In the future, we will continue further development of the signal processing to improve the sensitivity of the detector. Especially, the method to enhance the signal from the target is further considered, e.g., by integrating temporal domain processing into the detector.

\section{Data availability}

The data used in this research are provided by DENSO Corporation under a research and development agreement with Chemnitz University of Technology; therefore, the data are not publicly accessible.

Edited by: M. Chandra

Reviewed by: two anonymous referees

\section{References}

Cao, T. V., Palmer, J., and Berry, P. E.: False Alarm Control of CFAR Algorithms with Experimental Bistatic Radar Data, in: Proceedings of 2010 IEEE International Radar Conference, 1014 May 2010, Arlington, USA, 156-161, 2010.

Dickmann, J., Appenrodt, N., Klappstein, J., and Brenk, C.: Making Bertha See Even More: Radar Contribution, IEEE Access, 3, 1233-1247, doi:10.1109/ACCESS.2015.2454533, 2015.

Levinson, J., Askeland, J., Becker, J., Dolson, J., Held, D., Kammel, S., Kolter, J. Z., Langer, D., Pink, O., Pratt, V., Sokolsky, M., Stanek, G., Stavens, D., Teichman, A., Werling, M., and Thrun, S.: Towards Fully Autonomous Driving: Systems and Algorithms, in: Proceedings of 2011 IEEE Intelligent Vehicles Symposium, 5-9 June 2011, Baden-Baden, Germany, 163-168, 2011.

Ogawa, T., Sakai, H., Suzuki, Y., Takagi, K., and Morikawa, K.: Pedestrian detection and tracking using in-vehicle Lidar for automotive application, in: Proceedings of 2011 IEEE Intelligent Vehicles Symposium, 5-9 June 2011, Baden-Baden, Germany, 734-739, 2011.

Rohling, H.: Ordered statistic CFAR technique - an overview, in: Proceedings of 2011 12th International Radar Symposium (IRS), 7-9 September 2011, Leipzig, Germany, 631-638, 2011.

Rohling, H.: Fast Two-Dimensional CFAR Procedure, IEEE T. Aerosp. Elect. Syst., 49, 1817-1823, doi:10.1109/TAES.2013.6558022, 2013.

Rohling, H. and Mende, R.: OS CFAR performance in a $77 \mathrm{GHz}$ radar sensor for car application, in: Proceedings of 1996 CIE International Conference of Radar, 8-10 October 1996, Beijing, China, 109-114, 1996.

Ziegler, J., Bender, P., Schreiber, M., Lategahn, H., Strauss, T., Stiller, C., Dang, T., Franke, U., Appenrodt, N., Keller, C. G., Kaus, E., Herrtwich, R. G., Rabe, C., Pfeiffer, D., Lindner, F., Stein, F., Erbs, F., Enzweiler, M., Knöppel, C., Hipp, J., Haueis, M., Trepte, M., Brenk, C., Tamke, A., Ghanaat, M., Braun, M., Joos, A., Fritz, H., Mock, H., and Hein, M.: Making Bertha Drive - An Autonomous Journey on a Historic Route, IEEE Intel. Transport. Syst. Mag., 6, 8-20, doi:10.1109/MITS.2014.2306552, 2014a.

Ziegler, J., Lategahn, H., Schreiber, M., Keller, C. G., Knoppel, C., Hipp, J., Haueis, M., and Stiller, C.: Video based localization for Bertha, in: Proceedings of 2014 IEEE Intelligent Vehicles Symposium, 8-11 June 2014, Dearborn, MI, USA, 1231-1238, 2014b. 